\title{
APPENDIX A
}

\section{Supported Printer Manufacturers}

The following printer manufacturers have pre-loaded settings and profiles in MatterControl as of the writing of this book. If your manufacturer is not listed here, you might check to see if they were added more recently by looking in the printer configuration menus (see Chapter 3 ), or see if the manufacturer says MatterControl will work with your printer.

\section{D Factory}

3D Stuffmaker

Airwolf 3D

Blue Eagle Labs

Deezmaker

FlashForge (except Dreamer)

Leapfrog

Lulzbot

MakerGear

MAKEiT

MakerBot (prior to 5th Gen)

Me3D

OpenBeam

Portabee

Printrbot

PrintSpace

Revolution 3D Printers

ROBO 3D

SeeMeCNC

Solidoodle

Type A Machines

Velleman 